\title{
Superconductivity in the Ni-based ternary compound $\mathrm{La}_{6} \mathrm{Ni}_{2} \mathrm{Sn}$
}

\author{
J.W. Chen *, N.S. Jou, B.K. Wang, Ching-Ray Chang, R.C. Yang \\ Department of Physics, National Taiwan University, Taipei, Taiwan, ROC
}

Received 25 June 2002; received in revised form 31 July 2002; accepted 5 August 2002

\begin{abstract}
We have investigated the transport and magnetic properties of the ternary compound $\mathrm{La}_{6} \mathrm{Ni}_{2} \mathrm{Sn}$ by means of the ac electrical resistivity $\rho$, dc magnetic susceptibility $\chi_{\mathrm{dc}}$, and specific heat $C(T)$ measurements. Bulk superconductivity was observed in this compound with a superconducting transition temperature of $\sim 2.25 \mathrm{~K}$ as inferred from a sudden drop to zero value in the $\rho(T)$ curve, the diamagnetic behavior in the $\chi_{\mathrm{dc}}(T)$ curve, and a jump in the $C(T)$ curve. Type II superconductivity can also be inferred from the isothermal $M-H$ curve measured at $T=2 \mathrm{~K}$. The initial slope of the upper critical magnetic field $-\left(\mathrm{d} H_{\mathrm{c} 2} / \mathrm{d} T\right)_{T_{\mathrm{c} 0}}$ has a value of $4000 \mathrm{Oe} / \mathrm{K}$, from which a zero-temperature superconducting coherence length $\xi_{0}=230 \AA$ can be obtained. Analysis using the specific heat data yields a Debye temperature $\Theta_{\mathrm{D}}=135 \mathrm{~K}$ for this compound.
\end{abstract}

(C) 2002 Elsevier Science B.V. All rights reserved.

PACS: 74.25.Bt; 74.25.Fy; 74.25.Ha

\section{Introduction}

The discovery of superconductivity in the R$\mathrm{Ni}-\mathrm{B}-\mathrm{C}(\mathrm{R}=\mathrm{Y}$ or rare earth) systems has renewed a lot of research interest in the Ni-based compounds [1-3]. Studies of the rare-earth Nibased ternary compounds are of special interest because superconductivity and various types of magnetic order have been observed in these systems [4-7]. The $\mathrm{R}_{6} \mathrm{Ni}_{2} \mathrm{Sn} \quad(\mathrm{R}=$ rare earth ions) compounds are of the $\mathrm{Ho}_{6} \mathrm{Ni}_{2} \mathrm{Ga}$-type structure with space group Immm [8]. Among the $\mathrm{R}_{6} \mathrm{Ni}_{2} \mathrm{Sn}$ compounds, $\mathrm{Er}_{6} \mathrm{Ni}_{2} \mathrm{Sn}$ has been mostly studied

\footnotetext{
${ }^{*}$ Corresponding author. Tel.: +886-2-33665172; fax: +886-223639984.

E-mail address: jwchen@phys.ntu.edu.tw (J.W. Chen).
}

because of its usage for the magnetic refrigeration $[9,10]$. In this paper, we report the first observation of bulk superconductivity in the Ni-based ternary compound $\mathrm{La}_{6} \mathrm{Ni}_{2} \mathrm{Sn}$ with a transition temperature of $2.25 \mathrm{~K}$. In addition, isothermal magnetization measurements at $T=2 \mathrm{~K}$ reveal type II superconductivity in this compound.

\section{Experimental}

The polycrystalline samples of $\mathrm{La}_{6} \mathrm{Ni}_{2} \mathrm{Sn}$ were prepared by arc-melting stoichiometric amounts of the constituent elements (La: $99.99 \%$, Ni: $99.99 \%$, Sn: $99.9999 \%$ ) together on a water-cooled copper hearth in a Zr-gettered argon atmosphere. A twostage procedure $\left(\mathrm{La}_{6}+\mathrm{Ni}_{2} \mathrm{Sn}\right)$ was utilized with negligible weight loss $(<0.5 \%)$. To improve the 
sample homogeneity, the as melted samples were subsequently wrapped in Ta foil, sealed in quartz tube in argon atmosphere, and annealed at $550{ }^{\circ} \mathrm{C}$ for 3 days. The ac electrical resistivity $\rho(T)$ measurements on bar-shaped samples were performed in a $\mathrm{He}^{4}$ cryostat between $300 \mathrm{~K}$ and $2 \mathrm{~K}$ using a four-wire ac impedance bridge operating at $16 \mathrm{~Hz}$ with silicon diode as the temperature sensor. The $\mathrm{dc}$ magnetic susceptibility measurements were performed in a commercial superconducting quantum interference device (SQUID) magnetometer from 2 to $300 \mathrm{~K}$ in various applied magnetic fields. The specific heat measurements were performed in a commercial calorimeter in the temperature range between 1.8 and $15 \mathrm{~K}$ in different applied fields $H$.

\section{Results and discussion}

The ac electrical resistivity $\rho$ versus temperature $T$ curve in zero magnetic field for $\mathrm{La}_{6} \mathrm{Ni}_{2} \mathrm{Sn}$ is plotted in Fig. 1(a) for $0 \leqslant T \leqslant 300 \mathrm{~K}$. Above the superconducting transition temperature, $\rho(T)$ curve exhibits typical characteristics of common metal and decreases monotonically with decreasing temperature with a RR ratio of 4.4 and a residue resistivity $\rho_{\mathrm{r}}=28.35 \mu \Omega \mathrm{cm}$. The relatively high value of the residue resistivity $\rho_{\mathrm{r}}$ indicates that the sample is probably not very homogeneous. At $\sim 2.8 \mathrm{~K}$, the resistivity starts to drop to zero and the sample becomes superconducting with the midpoint transition temperature $T_{\mathrm{c}}(50 \%)=2.4 \mathrm{~K}$ and a transition width $\Delta T_{\mathrm{c}}=0.58 \mathrm{~K}$ at zero magnetic field. Shown in Fig. 1(b) are selected curves for ac electrical resistivity $\rho$ as a function of temperature $T$ in different applied magnetic fields $H$ below $2.0 \mathrm{kOe}$. With increasing applied magnetic field, the transition curves shift to lower temperature and the transition widths increase. Finally, when the field gets higher than $2.0 \mathrm{kOe}$, the superconducting transition is lower than $2.0 \mathrm{~K}$.

The temperature dependences of the zero-field cooled (ZFC) and field-cooled (FC) magnetization curves measured in a field of 20 Oe between 2 and $4 \mathrm{~K}$ for the $\mathrm{La}_{6} \mathrm{Ni}_{2} \mathrm{Sn}$ sample are plotted in Fig. 2(a). The occurrence of diamagnetic transition in the ZFC curve near $2.4 \mathrm{~K}$ reveals the occurrence of superconductivity in this compound, in consistent
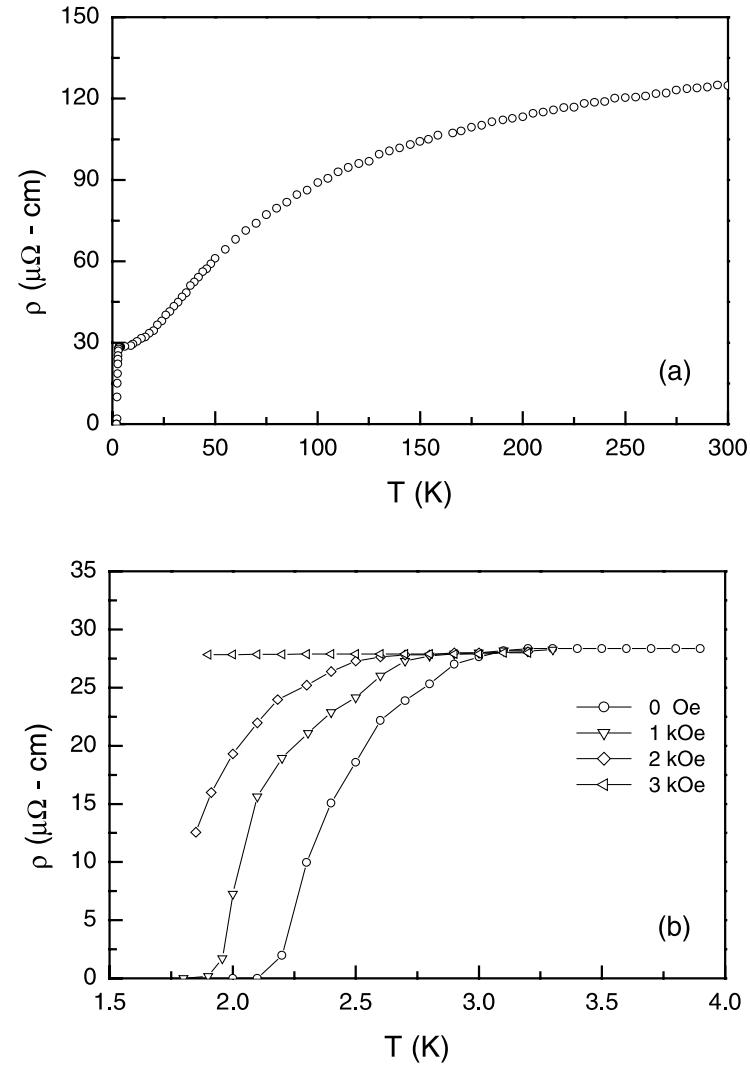

Fig. 1. (a) The ac electrical resistivity $\rho$ versus temperature $T$ curve in zero magnetic field for $\mathrm{La}_{6} \mathrm{Ni}_{2} \mathrm{Sn}$ for $0 \leqslant T \leqslant 300 \mathrm{~K}$. (b) $\rho(T)$ curves in different applied magnetic fields $H$ below 2.0 kOe.

with the result as obtained from the $\rho(T)$ curve. The diamagnetism is much weaker in the FC curve. Plotted in the inset of Fig. 2(a) is the temperature dependence of the magnetization of $\mathrm{La}_{6} \mathrm{Ni}_{2} \mathrm{Sn}$ measured in an applied field of $5 \mathrm{kOe}$. The very weakly temperature dependent susceptibility reveals that $\mathrm{La}_{6} \mathrm{Ni}_{2} \mathrm{Sn}$ is a Pauli paramagnet and there is no magnetic moment associate with the Ni ions. Fig. 2(b) shows the isothermal magnetization curve for $\mathrm{La}_{6} \mathrm{Ni}_{2} \mathrm{Sn}$ that was measured on increasing field at $T=2 \mathrm{~K}$. Which reveals type II superconductivity in this compound.

The specific heat as a function of temperature taken in zero magnetic field for $\mathrm{La}_{6} \mathrm{Ni}_{2} \mathrm{Sn}$ is plotted in Fig. 3(a) for $0 \leqslant T \leqslant 8 \mathrm{~K}$. The inset shows the details of the $C(T)$ curves in various applied magnetic fields (all data were taken with the 

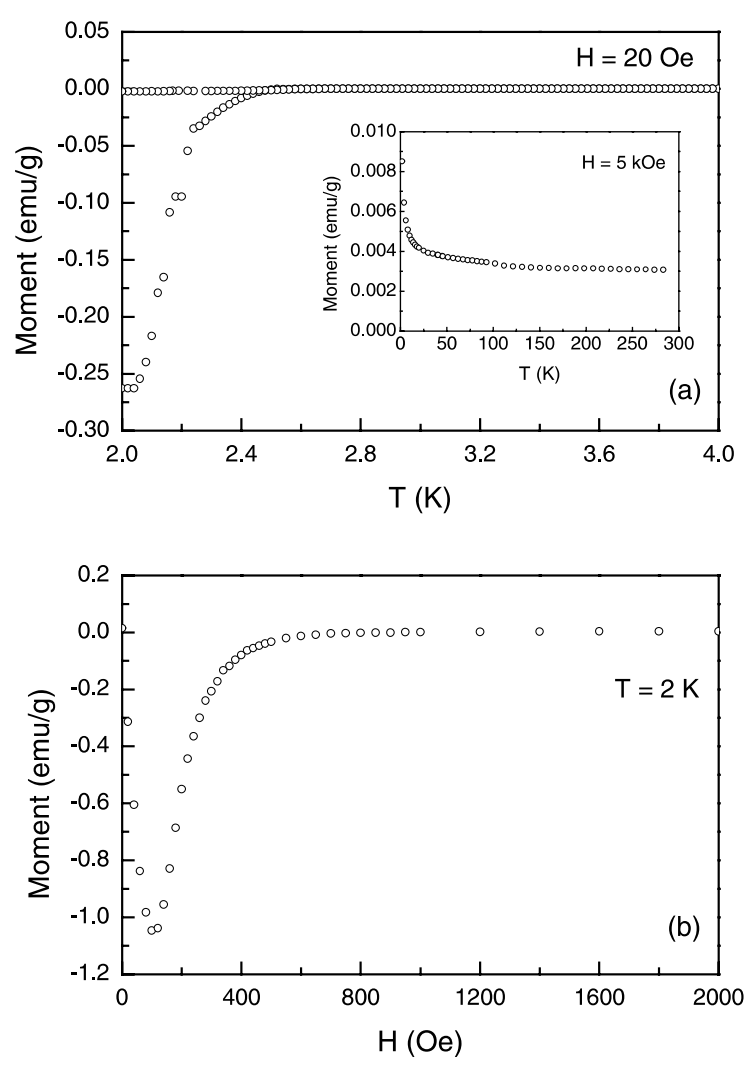

Fig. 2. (a) Temperature dependences of the ZFC and FC magnetization curves measured in a field of 20 Oe between 2 and $4 \mathrm{~K}$ for $\mathrm{La}_{6} \mathrm{Ni}_{2} \mathrm{Sn}$. The inset is the magnetization data measured in an applied field of $5 \mathrm{kOe}$. (b) The isothermal magnetization curve for $\mathrm{La}_{6} \mathrm{Ni}_{2} \mathrm{Sn}$ measured on increasing field at $T=2 \mathrm{~K}$.

magnetic field applied at the lowest temperature). Shown in Fig. 3(b) is the $C / T$ versus $T^{2}$ plot for this sample. The zero temperature value of the electronic specific heat coefficient $\gamma=6.30 \mathrm{~mJ} / \mathrm{mol}$ $\mathrm{La}-\mathrm{K}^{2}$ is obtained and the value of $\beta$ as obtained from the slope at low temperatures is $1.19 \mathrm{~mJ} / \mathrm{mol}$ $\mathrm{La}-\mathrm{K}^{4}$. The latter value corresponds to the Debye temperature $\Theta_{\mathrm{D}}=135 \mathrm{~K}$ for this compound. The observed value of $\Delta C / \gamma T_{\mathrm{c}}$ at zero field is about 0.66 , which is smaller than 1.43 as predicted from the BCS theory. Nevertheless, the discontinuity in the $C(T)$ curve is in consistent with the results of $\rho(T)$ and $\chi(T)$ measurements and indicates that $\mathrm{La}_{6} \mathrm{Ni}_{2} \mathrm{Sn}$ undergoes bulk superconducting transition with $T_{\mathrm{c}} \sim 2.25 \mathrm{~K}$. Works for improv-
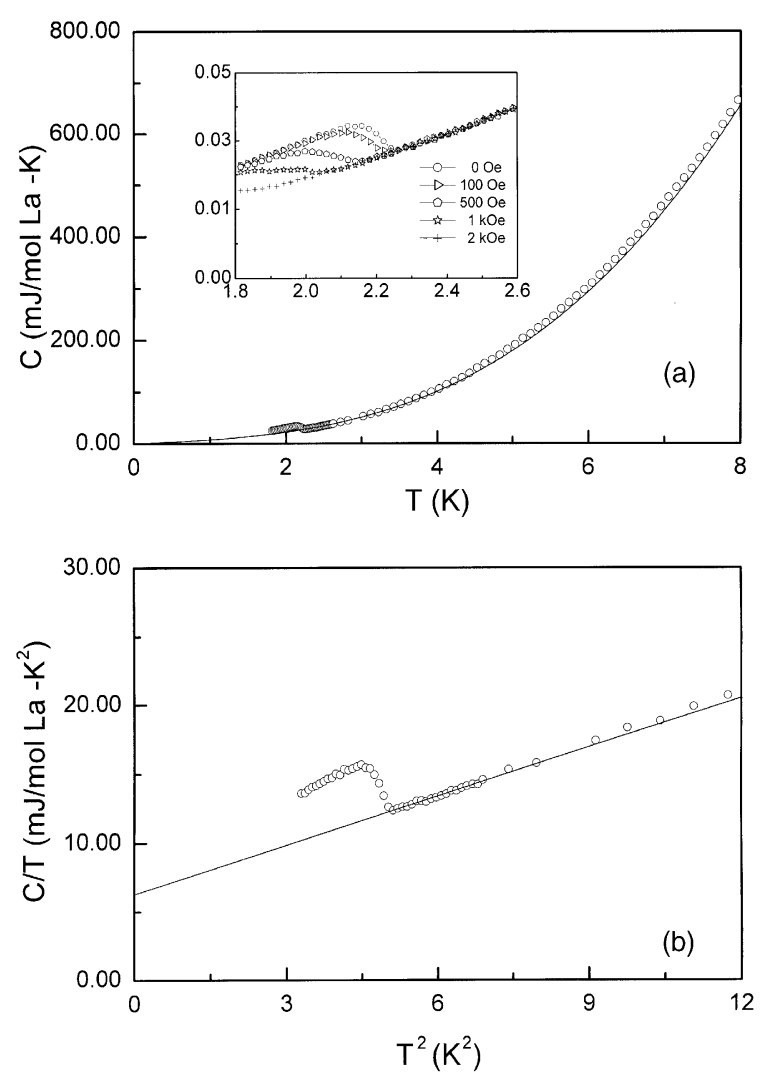

Fig. 3. (a) The specific heat as a function of temperature taken in zero magnetic field for $\mathrm{La}_{6} \mathrm{Ni}_{2} \mathrm{Sn}$ for $0 \leqslant T \leqslant 8 \mathrm{~K}$, with the inset showing the details of the $C(T)$ curves in various applied magnetic fields. (b) $C / T$ versus $T^{2}$ plot for $\mathrm{La}_{6} \mathrm{Ni}_{2} \mathrm{Sn}$.

ing the sample homogeneity are currently in progress.

The upper critical field $H_{\mathrm{c} 2}(T)$ for $\mathrm{La}_{6} \mathrm{Ni}_{2} \mathrm{Sn}$ cannot be obtained because it requires measurements far below $1.8 \mathrm{~K}$ (the lowest temperature we can achieve). However, one can estimate the orbital critical magnetic field at zero temperature from the weak-coupling formula [11] $H_{\mathrm{c} 2}^{*}(0)=$ $-0.693 T_{\mathrm{c} 0}\left(\mathrm{~d} H_{\mathrm{c} 2} / \mathrm{d} T\right)_{T_{\mathrm{c} 0}}$. The initial slope of the upper critical magnetic field $-\left(\mathrm{d} H_{\mathrm{c} 2} / \mathrm{d} T\right)_{T_{\mathrm{c} 0}}$ has a value of $4000 \mathrm{Oe} / \mathrm{K}$ (data not shown here), and the obtained value of $H_{\mathrm{c} 2}^{*}(0)$ is $\sim 6240$ Oe. From this, the coherence length at zero temperature for this compound can be estimated from the relation [12] $\xi_{0}^{2}=\Phi_{0} / 2 \pi H_{\mathrm{c} 2}^{*}(0)$, where $\Phi_{0}=c h / 2 e=2.07 \times 10^{-7}$ $\mathrm{Oe} \mathrm{cm}^{2}$ is the flux quantum. The obtained value of $\xi_{0}$ is $\sim 230 \AA$. 
The value of the transport mean free path $l_{\text {tr }}$ for $\mathrm{La}_{6} \mathrm{Ni}_{2} \mathrm{Sn}$ can be estimated from the normal state resistivity at low temperatures by means of the formula [13] $l_{\mathrm{tr}}=\left(1.27 \times 10^{4}\right) / \rho_{0}(Z / \Omega)^{2 / 3}$, where $l_{\text {tr }}$ is in $\mathrm{cm}, \rho_{0}$ is in $\Omega \mathrm{cm}$, and $(Z / \Omega)$ is the density of the conduction electrons. As a rough approximation, we assume the conduction electrons are contributed by the trivalent La atoms. And the value of $(Z / \Omega) \sim 8.42 \times 10^{22} \mathrm{~cm}^{-3}$ is obtained. In addition, the extrapolated zero temperature resistivity $\rho_{0}$ for this sample can be replaced by the residue resistivity $\rho_{\mathrm{r}}$, which has a value of $\rho_{0}=28.35 \mu \Omega \mathrm{cm}$. The value of $l_{\text {tr }} \sim 23 \AA$ is then obtained. This value is considerably smaller than that of $\xi_{0}$, suggesting that $\mathrm{La}_{6} \mathrm{Ni}_{2} \mathrm{Sn}$ is a dirty superconductor.

The conduction electron effective mass $m_{\text {eff }}$ for $\mathrm{La}_{6} \mathrm{Ni}_{2} \mathrm{Sn}$ can be calculated from the equation [14] $m_{\text {eff }}=h^{2} k_{\mathrm{F}}^{2} \gamma / 4 \pi^{4}(Z / \Omega) k_{\mathrm{B}}^{2}$. Here $\gamma$ is the electronic specific heat coefficient for $\mathrm{La}_{6} \mathrm{Ni}_{2} \mathrm{Sn}$ which has a value of $6.30 \mathrm{~mJ} / \mathrm{mol} \mathrm{La}-\mathrm{K}^{2}$ as obtained from the $C(T)$ measurement. The Fermi wave vector $k_{\mathrm{F}}$ can be estimated (with the assumption of a spherical Fermi surface in the sample) from the relation $k_{\mathrm{F}}=\left(3 \pi^{2} Z / \Omega\right)^{1 / 3}$ and yields a value of $k_{\mathrm{F}}=1.36 \times$ $10^{8} \mathrm{~cm}^{-1}$. From this, we then obtain $m_{\mathrm{eff}}=2.4 m_{\mathrm{e}}$, where $m_{\mathrm{e}}$ is the mass for the free electron.

In summary, we have performed the ac electrical resistivity $\rho$, dc magnetic susceptibility $\chi_{\mathrm{dc}}$, and specific heat $C(T)$ studies on $\mathrm{La}_{6} \mathrm{Ni}_{2} \mathrm{Sn}$. We found that is a type II superconductor with $T_{\mathrm{c}}=2.25 \mathrm{~K}$ and $-\left(\mathrm{d} H_{\mathrm{c} 2} / \mathrm{d} T\right)_{T_{\mathrm{c} 0}}=4000$ Oe/K. The obtained value of transport mean free path $l_{\text {tr }}$ is $23 \AA$, which is much smaller than the value of $\xi_{0}$ and implies dirty superconductivity in this compound. The Debye temperature for this compound is about 135 $\mathrm{K}$ and the effective mass of the conduction electrons is about $2.4 m_{\mathrm{e}}$ as estimated from the $C(T)$ data.

\section{Acknowledgement}

This work was supported by the ROC National Science Council under grant no. NSC 90-2112M002-042.

\section{References}

[1] R. Nagarajan, C. Mazumdar, Z. Hossain, S.K. Dhar, K.V. Gopalakrish, L.C. Gupta, C. Godart, B.D. Padalia, R. Vijayaraghavan, Phys. Rev. Lett. 72 (1994) 274.

[2] R.J. Cava, H. Takagi, H.W. Zundbergen, J.J. Krajewski, W.F. Peck Jr., T. Siegrist, B. Battogg, R.B. van Dover, R.J. Felder, K. Mizuhashi, J.O. Lee, H. Eisaki, S. Uchida, Nature (London) 367 (1994) 252.

[3] T. Siegrist, H.W. Zundbergen, R.J. Cava, J.J. Krajewski, W.F. Peck Jr., Nature (London) 367 (1994) 254.

[4] W.H. Lee, H.K. Zeng, Y.D. Yao, Y.Y. Chen, Physica C 266 (1996) 138.

[5] W. Schäfer, W. Kockelmann, G. Will, J.K. Yakinthos, P.A. Kotsanidis, J. Alloys Comp. 250 (1997) 565.

[6] K. Motoya, K. Nakaguchi, N. Kayama, K. Inari, J. Akimitsu, K. Izawa, T. Fujita, J. Phys. Soc. Jpn. 66 (1997) 1124.

[7] H. Onodera, Y. Koshikawa, M. Kosaka, M. Ohashi, H. Yamauchi, Y. Yamaguchi, J. Magn. Mag. Mat. 182 (1998) 161.

[8] O.M. Sichevich, L.P. Komarovskaya, Yu.N. Grin, Ya.P. Yarmolyuk, R.V. Skolozdra, Ukr. Fiz. J. 29 (1984) 1342.

[9] K.A. Gschneidner Jr., V.K. Pecharsky, M. Gailloux, in: R.G. Ross Jr. (Ed.), Crycollers 8, Plenum Press, New York, 1995.

[10] O. Syshchenko, Yu. Stadnyk, L. Romaka, Yu. Mudryk, R.V. Dremov, V. Sechovsky, J. Alloys Comp. 319 (2001) 14.

[11] R.R. Hake, Appl. Phys. Lett. 10 (1967) 186.

[12] M. Tinkham, Introduction to Superconductivity, McGraw-Hill, New York, 1975.

[13] T.P. Orlando, E.J. McNiff, S. Foner, M.R. Beasley, Phys. Rev. B 19 (1979) 4545.

[14] For example, see C. Kittel, Introduction to Solid State Physics, sixth ed., John Wiley \& Sons, New York, 1986. 